\title{
Elastic kirigami patch for electromyographic analysis of the palm muscle during baseball pitching
}

Kento Yamagishi ${ }^{1,2,9}$, Takenori Nakanishi ${ }^{2}$, Sho Mihara ${ }^{2,3}$, Masaru Azuma ${ }^{3}$, Shinji Takeoka ${ }^{3,4}$, Kazuyuki Kanosue ${ }^{5}$, Tomoyuki Nagami ${ }^{6}$ and Toshinori Fujie (i) ${ }^{1,7,8}$

\begin{abstract}
Surface electromyography (sEMG) is widely used to analyze human movements, including athletic performance. For baseball pitchers, a very precise movement is required to pitch the ball into the strike zone. The palm muscles appear to play a key role in this movement, and a real-time recording of sEMG from the palm muscle is useful in the analysis of motion during baseball pitching. However, the currently available devices with rigid and bulky electrodes (including connective wires) impede natural movements of the wearer and recording of sEMG from the palm muscles during vigorous action. Here, we describe a skin-contact patch consisting of kirigami-based stretchable wirings and conductive polymer nanosheet-based ultraconformable bioelectrodes, which address the challenge of mechanical mismatch between human skin and electrical devices. The key strategy is a kirigami-inspired wiring design and a mechanical gradient structure from nanosheet-based flexible bioelectrodes to a bulk wearable device. This approach would buffer the mechanical stress applied to the skin-contact bioelectrodes during an arm swing movement. With this patch, we precisely measure sEMG at the abductor pollicis brevis muscle (APBM) in a baseball player during ball pitching. We observe differences in the activity of the APBM between different types of pitches_-fastball and curveball. This sEMG measurement system will enable the analysis of motion in unexplored muscle areas, such as on the palm and the sole, leading to a deeper understanding of muscular activity during performance in a wide range of sports and other movements.
\end{abstract}

\section{Introduction}

Surface electromyography (sEMG) is widely used to analyze human movements, including athletic performance. Wearable devices that are minimally perceivable facilitate the accurate measurement of sEMG during exercise. Conventionally, sEMG has been recorded using small electrodes that are attached to the skin surface and connected to an amplifier with wires. However, these devices restrict free movement and produce artifacts

\footnotetext{
Correspondence: Tomoyuki Nagami (t-nagami@kitasato-u.ac.jp) or

Toshinori Fujie (t_fujie@bio.titech.ac.jp)

${ }^{1}$ Research Organization for Nano \& Life Innovation, Waseda University, Tokyo, Japan

${ }^{2}$ Graduate School of Advanced Science and Engineering, Waseda University, Tokyo, Japan

Full list of author information is available at the end of the article.
}

during vigorous movements. Recent technological advances enabling small all-in-one skin-contact modules containing electrodes and amplifiers with telemetric signal transmission have addressed the above-mentioned problems to some degree ${ }^{1-5}$. However, for some parts of the body, such as the palm, sole, or inner thigh, sEMG recording is still very difficult even with these small modules. For example, for baseball pitchers, the release of the ball requires a very precise movement, with only an $\sim 2 \mathrm{~ms}$ time window to pitch the ball into the strike zone. The palm muscles appear to play a key role in this movement ${ }^{6-10}$. Because the ball directly touches the palm muscles, sEMG recording from the palm muscles during an actual pitch is extremely difficult, and even the most recent recording modules cannot be utilized. Furthermore,

\section{(c) The Author(s) 2019}

(c) (i) Open Access This article is licensed under a Creative Commons Attribution 4.0 International License, which permits use, sharing, adaptation, distribution and reproduction cc) in any medium or format, as long as you give appropriate credit to the original author(s) and the source, provide a link to the Creative Commons license, and indicate if changes were made. The images or other third party material in this article are included in the article's Creative Commons license, unless indicated otherwise in a credit line to the material. If material is not included in the article's Creative Commons license and your intended use is not permitted by statutory regulation or exceeds the permitted use, you will need to obtain permission directly from the copyright holder. To view a copy of this license, visit http://creativecommons.org/licenses/by/4.0/. 
if electrodes are attached to the palm over the palm muscle, there is likely to be strain on the lead wires because the wrist flexes at a wide angle $\left(\sim 40^{\circ}\right)$, with a maximum angular velocity of $\sim 5000^{\circ} / \mathrm{s}$ during a pitch ${ }^{11}$. For the above reasons, sEMG analyses during baseball pitching have been restricted to the elbow ${ }^{12}$, scapular muscles ${ }^{13}$, and lower ${ }^{14}$ and upper extremities ${ }^{15}$, and no studies have examined sEMG activity in palm muscles during baseball release. In the present study, we addressed this problem by developing a skin-contact patch consisting of (1) conductive polymer nanosheet-based ultraconformable electrodes and (2) "kirigami"-based stretchable wiring.

Recent developments in flexible thin-film-type electronics have led to several skin-contact devices that can detect a range of biosignals ${ }^{1-5,16-18}$. However, these devices generally contain rigid metal wiring, electronic elements, and/or wireless modules with limited use on the palm due to discomfort derived from the mechanical mismatch between human skin and electrical devices ${ }^{19}$. Although printable elastic conductive inks containing metal nanoparticles and elastomers have been developed $^{20,21}$, printed nanocomposite wiring can modulate electrical properties such as conductivity according to strain, and this can cause noise artifacts in the recorded signal $^{22}$. Alternatively, while conductive fiber-based wiring has been developed as an alternative form of stretchable conductor for wearable devices ${ }^{23}$, the conductive fibers do not conformably adhere to the skin surface, thus resulting in poor signal quality during exercise ${ }^{24}$.

In this study, to address the mechanical mismatch between the palm and the sensing module (consisting of a battery, signal amplifier, and wireless transmitter), we developed a skin-contact patch-type electronic device consisting of stretchable wiring and conductive polymer nanosheet-based bioelectrodes. The key strategy was a kirigami-inspired wiring design and a mechanical gradient structure ${ }^{25}$ from nanosheet-based flexible bioelectrodes to a bulk wearable device. We anticipated that this approach would buffer the mechanical stress applied to the skin-contact bioelectrodes during the arm swing movement. "Kirigami"-a type of Japanese paper art, referred to as cutting ("kiri-") paper ("-gami")-has been widely employed in the field of stretchable electronics because this technique renders unstretchable and rigid two-dimensional (2D) materials such as graphene ${ }^{26}$ and carbon nanotube-based nanocomposites ${ }^{27}$ stretchable via three-dimensional (3D) deformation ${ }^{28}$. Recent studies have enclosed metal wires within kirigami-patterned insulating materials (such as parylene and silicone rubbers) for application as stretchable neural probes ${ }^{29}$ or wearable devices ${ }^{30}$. As a connective section between nanosheet-based bioelectrodes and a bulk wearable module, we designed and developed a kirigami-based wiring system possessing the following features: (i) 2D-membrane-based conformable adhesion to the skin, (ii) stretchability with minimal changes in resistance, and (iii) a fully insulated structure containing a pantograph-like conductive layer and kirigami-patterned elastomeric insulating layers. We assembled conductive nanosheet-based ultraconformable bioelectrodes and a kirigami-based stretchable wiring system to form a patchtype skin-contact device that we termed the "elastic kirigami patch" (Fig. 1a). Using this device, we succeeded in the precise measurement of sEMG signals from the abductor pollicis brevis muscle (APBM), the largest muscle of the thenar eminence muscle group, during pitching in an experienced baseball player. For further analysis, we synchronized the obtained sEMG signals as well as the acceleration of the arm with sequential photographs of the pitching motion recorded using high-speed cameras (Fig. 1b).

\section{Materials and methods Materials}

A poly(ethylene terephthalate) (PET) film substrate (Lumirror 25T60) was purchased from Panac Co. (Tokyo, Japan). A $25-\mu \mathrm{m}$-thick polyimide film (Kapton ${ }^{\circledR}$ polyimide film) was purchased from DuPont (Wilmington, DE). A $200-\mu \mathrm{m}$-thick silicone rubber sheet was purchased from Asahi Rubber Inc. (Saitama, Japan). A poly(3,4-ethylene dioxythiophene):poly(styrene sulfonate) (PEDOT:PSS) aqueous dispersion, Clevios PH 1000 (1:2.5 PEDOT:PSS ratio), was purchased from H.C. Starck GmbH (Leverkusen, Germany). Poly(vinyl alcohol) (PVA, $\mathrm{Mw}=\sim 22,000$ ), ethyl acetate, and tetrahydrofuran (THF) were purchased from Kanto Chemical Co. (Tokyo, Japan). Polystyreneblock-polybutadiene-block-polystyrene (SBS; $\mathrm{Mw}=\sim 140$ $\mathrm{kDa}$ ) and Zonyl ${ }^{\circledR}$ FS-300 fluorosurfactant (zonyl) were purchased from Sigma-Aldrich, Co. (St. Louis, MO). Dipropylene glycol (DPG) was purchased from Tokyo Chemical Industry Co. (Tokyo, Japan).

\section{Preparation of conductive nanosheets and transfer to the skin}

The PEDOT:PSS/SBS conductive nanosheet was fabricated by a roll-to-roll gravure printing technique ${ }^{31}$. First, a water-soluble sacrificial layer was formed on a PET substrate by gravure-coating of a PVA aqueous solution (polymer concentration: $20 \mathrm{mg} / \mathrm{mL}$ ). On the dried PVA sacrificial layer, an SBS solution in THF (polymer concentration: $20 \mathrm{mg} / \mathrm{mL}$ ) was gravure-printed to form an SBS nanosheet with a thickness of $171 \pm 20 \mathrm{~nm}$. Next, the PEDOT:PSS aqueous dispersion (Clevios PH 1000) was mixed with DPG $(5 \mathrm{v} / \mathrm{v} \%)$ and zonyl $(1 \mathrm{v} / \mathrm{v} \%)$ for $8 \mathrm{~h}$ at room temperature and gravure-printed over the SBS layer to form a PEDOT:PSS nanosheet with a thickness of $\sim 168 \mathrm{~nm}$. A thermal treatment $\left(140^{\circ} \mathrm{C}, 15 \mathrm{~min}\right)$ was carried out on the rolled sample (width: $12 \mathrm{~cm}$; length: $\sim 5 \mathrm{~m}$ ) to confer water resistance to the PEDOT:PSS layer and enhance the conductivity. By making an adhesive tape frame on the top of PEDOT:PSS layer, the PEDOT:PSS/ 


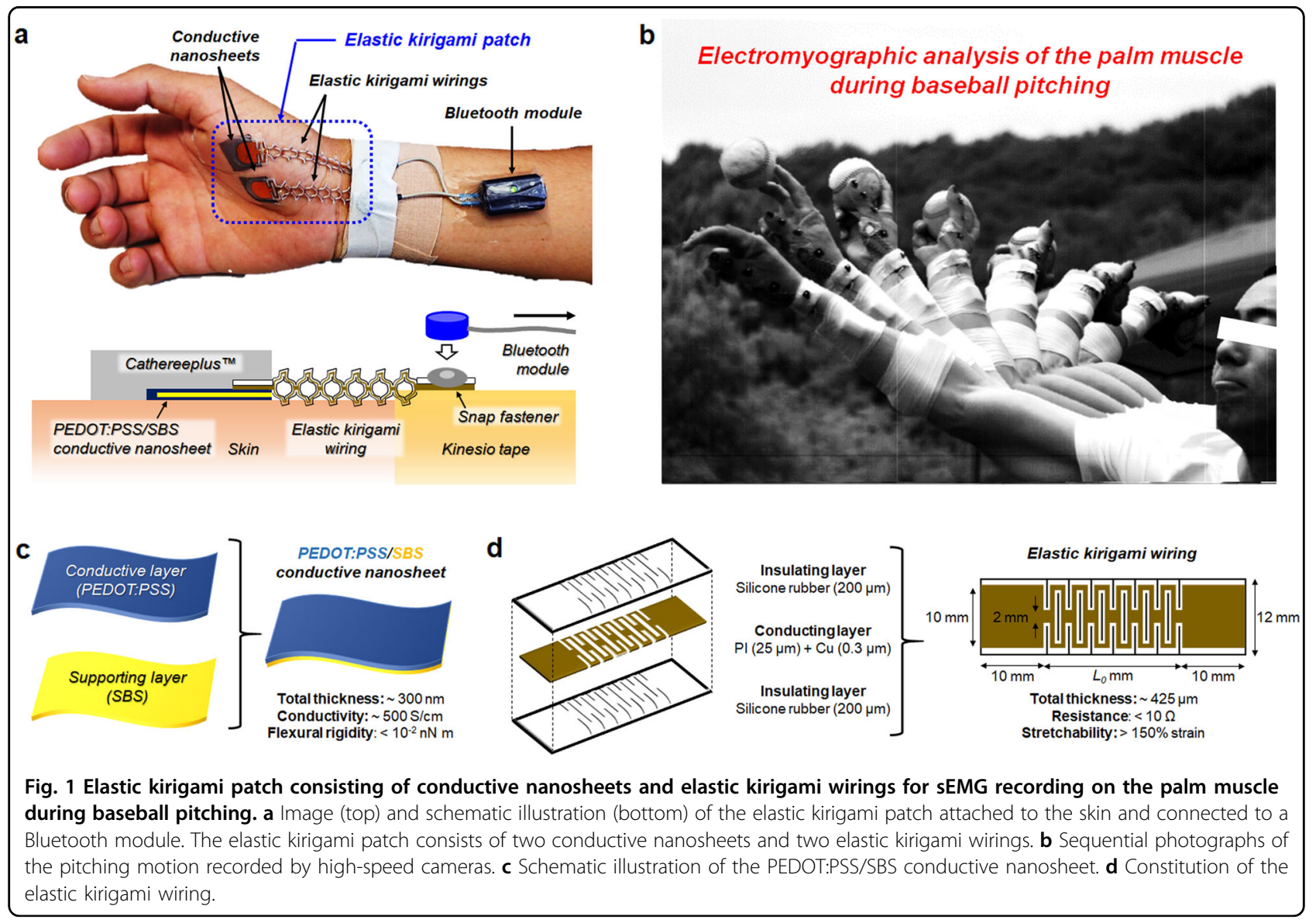

SBS/PVA tri-layered film was physically peeled off from the PET substrate. With a mechanical support with the tape frame, a free-standing PEDOT:PSS/SBS bilayered conductive nanosheet (total thickness: $339 \pm 91 \mathrm{~nm}$ ) was obtained by dissolving the PVA layer in water. Then, the nanosheet was folded into two layers by attaching the SBS layer to expose PEDOT:PSS on both the top and bottom layers and then collected on a nylon mesh as a temporary substrate. The tape frame was cut and removed, followed by transferring the nanosheet to the skin (APBM) with the aid of a small amount of water.

\section{Preparation of elastic kirigami wirings}

A Cu-sputtered polyimide film was prepared by sputtering $\mathrm{Cu}$ on a $25-\mu$ m-thick polyimide film (Kapton ${ }^{\circledast}$ polyimide film, DuPont) for 1,3 , or 5 min with a sputtering machine (SPT-4STD; Toei Scientific Industrial Co., Ltd, Miyagi, Japan). The $\mathrm{Cu}$-sputtered polyimide film and a $200-\mu \mathrm{m}$ thick silicone rubber sheet were cut into a multipantographlike or a kirigami pattern with a laser cutting machine (LaserPro Spirit SI-30; GCC, New Taipei City, Taiwan). Before sandwiching the multipantograph-patterned $\mathrm{Cu}$ sputtered polyimide film between two kirigami-patterned silicone rubber sheets, the corona treatment was carried out on the surface of the silicone rubber sheet with a corona treater (Corona Master PS-1M; Shinko Electric \& Instrumentation Co., Ltd, Osaka, Japan) to achieve permanent bonding between the top and bottom silicone rubber layers. After overlaying the three layers with an alignment by visual inspection, thermocompression bonding was applied to integrate the sandwiched structure with a handmade heat crimping machine for $5 \mathrm{~min}$ at $80^{\circ} \mathrm{C}$.

\section{Mechanical and electrical characterization of elastic kirigami wirings}

The stress-strain (s-s) curves of elastic kirigami wirings, a bare kirigami wiring, a nonkirigami wiring, and a silicone rubber sheet (Figs. 2a and 3b) were obtained by a standard tensile test using a tensile tester (EZ-S-5N; Shimadzu Co., Ltd, Kyoto, Japan) at a tensile rate of $10 \mathrm{~mm} / \mathrm{min}$. The samples were elongated until they ruptured. To obtain the $\mathrm{s}-\mathrm{s}$ curve shown in Fig. 3c, the sample was elongated until reaching $150 \%$ strain at a tensile rate of $10 \mathrm{~mm} / \mathrm{min}$ and kept in that state for $1 \mathrm{~min}$; then, it contracted to the initial length ( $0 \%$ strain) at the same tensile rate. To obtain the $R /$ $R_{0}$-strain curves shown in Fig. 3a, c and e, the resistance of the samples was measured in real time during elongation and contraction using a digital multimeter (PC720M; Sanwa 

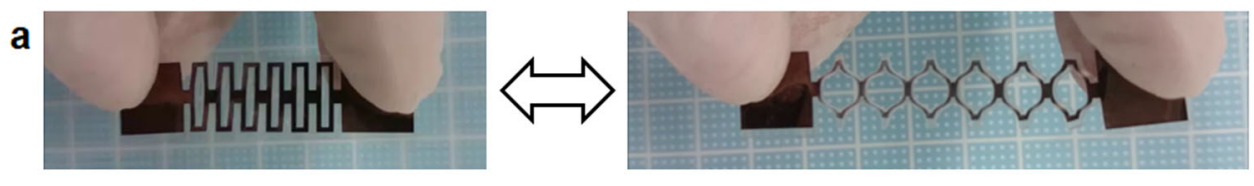

b

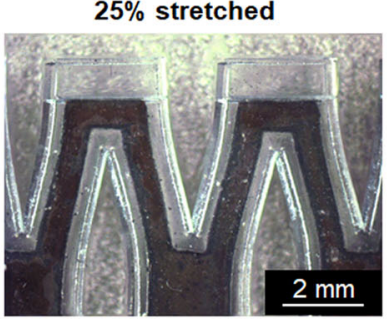

$100 \%$ stretched

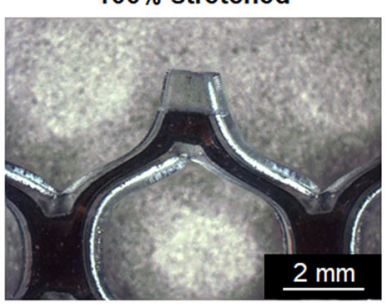

$150 \%$ stretched

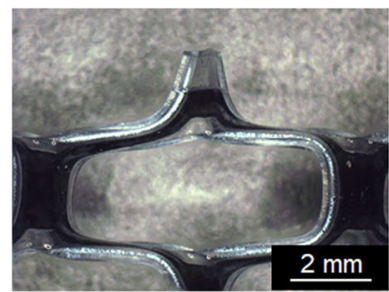

C
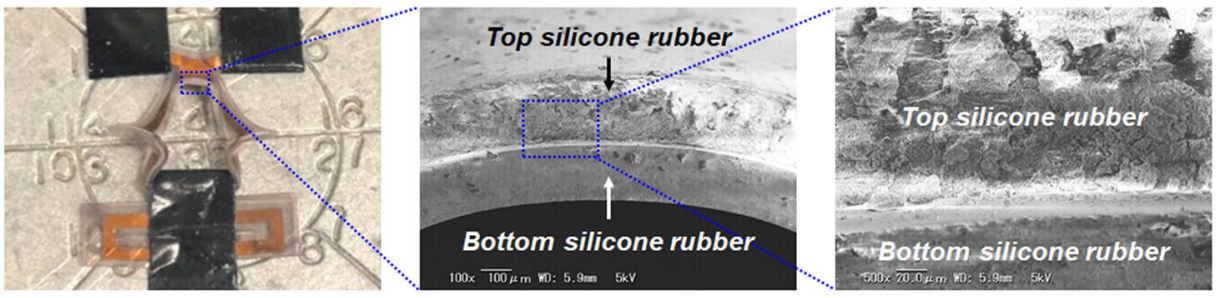

d
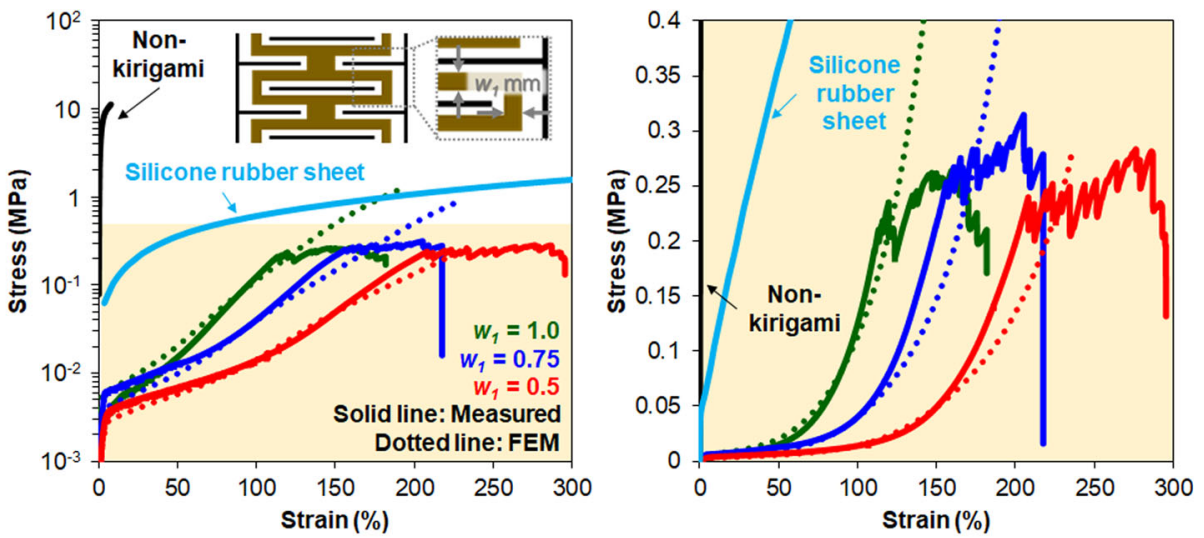

e $0 \%$

$50 \%$
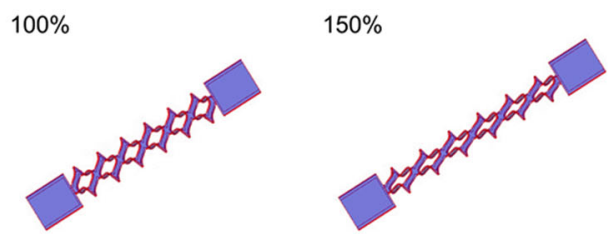

Fig. 2 Mechanical properties of elastic kirigami wirings. a Images of the elastic kirigami wiring before (left) and after (right) stretching by hand force. b Microscopic images of the elastic kirigami wiring elongated at 25\% (left), 100\% (middle), and 150\% (right) strain. c Optical (left) and SEM images (middle and right) of the elastic kirigami wiring under 150\% strain. There is no delamination between the top and bottom silicone rubber layers. $\mathbf{d}$ Stress-strain curves of the elastic kirigami wirings of three different designs, the nonkirigami sample, and the silicone rubber sheet (solid lines: measured, dotted lines: FEM-simulated). Left and right graphs are shown in exponential and linear scales of stress, respectively. e FEM-simulated images of the elastic kirigami wiring of $w_{1} / w_{2} / W_{3} / w_{4}=0.75 / 3.5 / 0.5 / 1.0$ at $0 \%, 50 \%, 100 \%$, and $150 \%$ (from left to right) tensile strain.

Electric Instrument Co., Ltd, Tokyo, Japan) connected to a data logger (PC Link 7; Sanwa).

\section{D finite element method simulation}

The finite element method (FEM) simulation for the s-s curves and the 3D elongation model of elastic kirigami wirings was performed using simulation software (Femtet;
Murata Software Co., Ltd, Tokyo, Japan). The structure (thickness, length, and pattern) of the sample was set as the actual value. The Young's moduli $(E)$ and Poisson ratios $(v)$ of $\mathrm{Cu}$-sputtered polyimide film $(\mathrm{Cu}-\mathrm{PI})$ and a silicone rubber sheet (SR) were set as $E_{\mathrm{Cu}-\mathrm{PI}}=3.4 \mathrm{GPa}$, $E_{\mathrm{SR}}=2.29 \mathrm{MPa}, v_{\mathrm{Cu}-\mathrm{PI}}=0.3$, and $v_{\mathrm{SR}}=0.49$, respectively. The mesh size of the simulation was $0.4 \mathrm{~mm}$. 

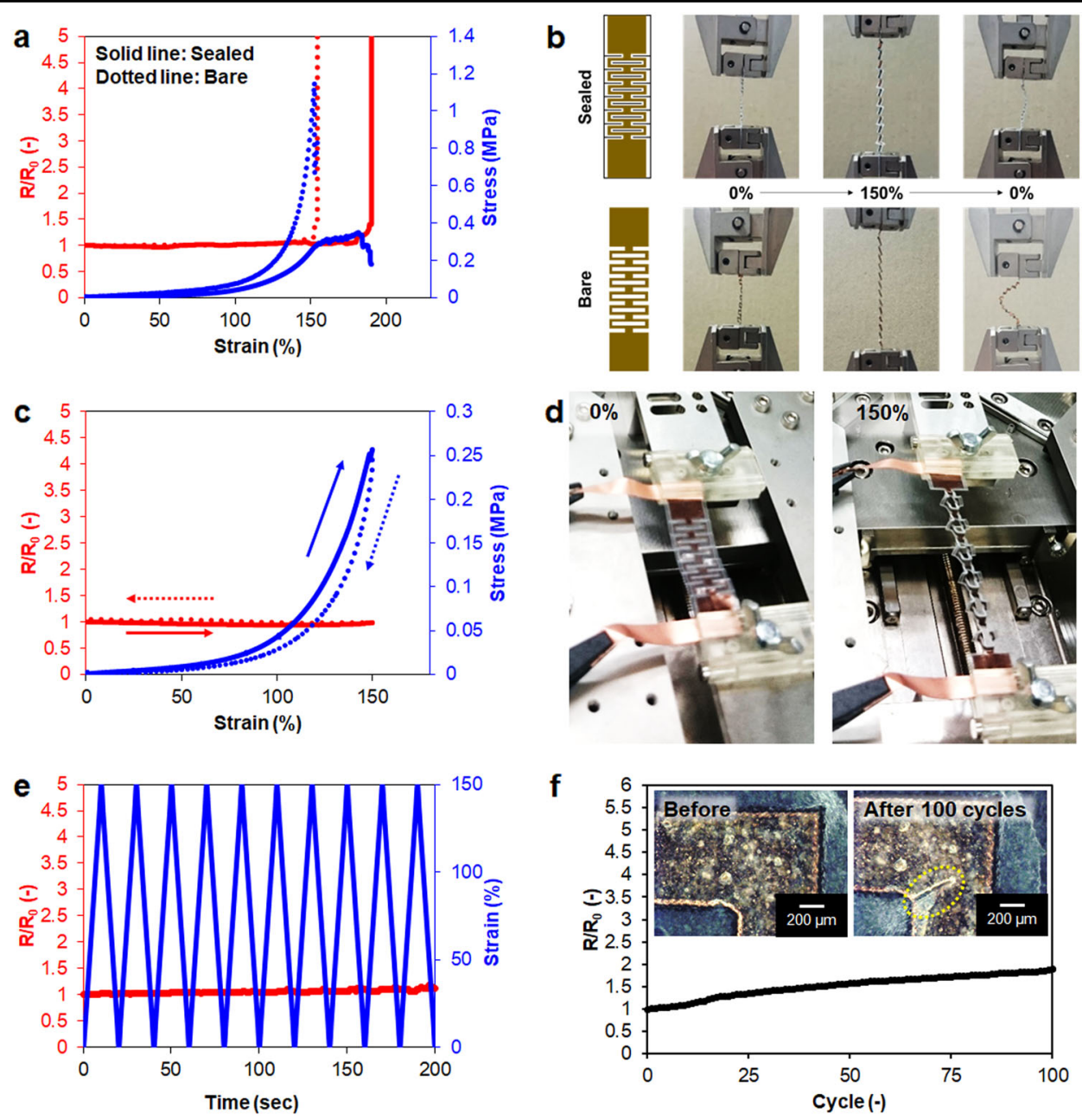

Fig. 3 Electrical properties of elastic kirigami wirings. a The relationship between the applied tensile strain and the normalized resistance $R / R_{0}$ of the wirings (red lines) or the loaded tensile stress (blue lines). $\mathbf{b}$ Illustrations and images of the sealed and bare samples while they were stretched by a tensile tester from the original length to $150 \%$ strain and then back to $0 \%$ strain. c The relationship between the applied tensile strain and the normalized resistance $R / R_{0}$ of the elastic kirigami wiring (red line) or the loaded tensile stress (blue line) while it was stretched by a tensile tester from the original length to $150 \%$ strain (solid line) and then back to $0 \%$ strain (dotted line). $\mathbf{d}$ Images of the elastic kirigami wiring elongated from $0 \%$ (left) to $150 \%$ strain (right) by a handmade tensile device. e Change in the normalized resistance $R / R_{0}$ of the elastic kirigami wiring (red line) and the applied tensile strain (blue line) against repeated cycles (the initial 10 cycles are shown). $\mathbf{f}$ The relationship between the standard resistance $\left(R / R_{0}\right)$ of the elastic kirigami wiring and the number of cycles of elongation (until 150\% strain) and contraction (until 0\% strain). Optical microscopic images of the elastic kirigami wiring before (left) and after 100 cycles of stretching and contracting (right). A crack in the Cu layer was observed on the corner of the wiring in the right image (see yellow circle).

\section{Installation of elastic kirigami patch on the palm skin}

Two sheets of conductive nanosheets were transferred onto the palm skin (APBM) of the subject in the manner described above. Next, two elastic kirigami wirings were placed on a sheet (size: $30 \mathrm{~mm} \times 50 \mathrm{~mm}$ ) of polyurethanebased transparent adhesive plaster (Cathereeplus ${ }^{\mathrm{TM}}$, Nichiban Co., Ltd, Tokyo). In this case, the $\mathrm{Cu}$ layer was exposed by removing the silicone rubber layer in the edge of the wirings ( $10 \mathrm{~mm}$ from the edge), and the opposite side of the exposed $\mathrm{Cu}$ layer was attached to the adhesive layer of Cathereeplus ${ }^{\mathrm{TM}}$. The elastic kirigami wiring-laden Cathereeplus $^{\mathrm{TM}}$ was attached to the palm skin to obtain an electrical connection between the conductive nanosheet electrodes and exposed $\mathrm{Cu}$ layer of wirings. The other edge of the wirings, where snap fasteners were equipped, was connected to the lead wirings of the Bluetooth module via the snap fasteners. The connection part and the Bluetooth module were fixed to the skin by covering a sheet of Cathereeplus ${ }^{\mathrm{TM}}$ and by rolling elastic therapeutic tape (Kinesio tape) around the arm.

\section{Stability of conductive nanosheets on the palm muscle}

The mechanical and electrical stability of the PEDOT: PSS/SBS nanosheet on the palm muscle against repetitive 
mechanical strain, i.e., stretching and contracting of the muscle, were evaluated by using a rectangular-shaped nanosheet $(1.5 \mathrm{~cm} \times 3 \mathrm{~cm})$ transferred to the palm muscle of one subject. The electrical contact with nanosheets was provided by two sheets of Au-sputtered polyimide thin films (thickness: $13 \mathrm{~mm}$ ) placed at both ends of the nanosheet. After the nanosheet and Au-sputtered polyimide thin films were covered with Cathereeplus ${ }^{\mathrm{TM}}$ $(3 \mathrm{~cm} \times 5 \mathrm{~cm})$, the resistance of the nanosheet was measured at the initial and most contracted/stretched states of the palm muscle. The measurements were repeated for five cycles of the three different states.

\section{Measurement of electrode-skin contact impedance}

Two sheets of conductive nanosheets and elastic kirigami wiring-laden Cathereeplus ${ }^{\mathrm{TM}}$ were attached to the palm muscle (APBM) in the same manner described above. The other edge of the wirings, where the snap fasteners were equipped, was connected to a chemical impedance meter (HIOKI 3532-80; HIOKI E.E. Co., Nagano, Japan). The impedance was measured in the frequency range from $10 \mathrm{~Hz}$ to $100 \mathrm{kHz}$.

\section{Analysis of signal-to-noise ratio from obtained sEMG signals}

The signal-to-noise ratio (SNR) was calculated by taking the highest sEMG signal peak $\left(A_{s}\right)$ during the active period and the standard deviation (STD) of the background $\left(A_{n}\right)$ during the inactive period. From these values, the SNR was calculated by the following formula ${ }^{32}$ :

$$
\mathrm{SNR}(\mathrm{dB})=20 \log _{10} \frac{A_{s}}{A_{n}} .
$$

\section{Results and discussion}

Preparation of conductive nanosheets and attachment to the skin on the palm

To measure sEMG signals from the palm in a way that is minimally perceivable to the wearer of the device, we used conductive polymer ultrathin films based on poly(3,4ethylene dioxythiophene):poly(styrene sulfonate) (PEDOT: PSS), referred to as "conductive nanosheets" 32 , as ultraconformable skin-contact electrodes. We previously investigated the mechanical and electrical stability of the PEDOT:PSS-based conductive nanosheets against sweat and found that they retained their proper electrical functions with structural integrity against immersion in artificial sweat solutions at $\mathrm{pH} 5.5$ and $\mathrm{pH} 8.0$ for at least $180 \mathrm{~min}^{31}$. In addition, our group demonstrated that bilayered elastic conductive nanosheets consisting of PEDOT:PSS and polystyrene-polybutadiene-polystyrene triblock copolymer (SBS) conformably adhered to human skin without any adhesive reagents and that they did not interfere with the natural deformation of skin because they have low flexural rigidity $\left(<10^{-2} \mathrm{nNm}\right)^{33}$. In this study, we prepared a PEDOT:PSS/SBS bilayered conductive nanosheet with a thickness of $339 \pm 91 \mathrm{~nm}$ (PEDOT:PSS layer: $\sim 168 \mathrm{~nm}$, SBS layer: $\sim 171 \mathrm{~nm}$ ), a conductivity of $\sim 500 \mathrm{~S} / \mathrm{cm}$, and a flexural rigidity of less than $10^{-2} \mathrm{nN} \mathrm{m}$ (Fig. 1c) using a gravure-coating-based roll-to-roll method ${ }^{31}$. To obtain a free-standing film, we employed a water-soluble PVAassisted sacrificial layer method (see Methods section and Fig. S1 for details). The flexibility (Young's modulus: $\sim 6 \mathrm{MPa}$, flexural rigidity: $\sim 10^{-5} \mathrm{nNm}$ ), stretchability (elongation: $\sim 150 \%$ ), and robustness (maximum tensile strength: $\sim 1.0 \mathrm{MPa}$ ) of the SBS nanosheet ${ }^{33,34}$ enabled the bilayered PEDOT:PSS/SBS conductive nanosheet to conform to van-der-Waals-force-based conformable adhesion to the skin without the use of any adhesive agents.

\section{Stability of conductive nanosheets on the palm muscle}

We evaluated the mechanical and electrical stability of the PEDOT:PSS/SBS nanosheet on the palm muscle against repetitive mechanical strain, i.e., stretching and contracting of the muscle. A rectangular-shaped nanosheet $(1.5 \mathrm{~cm} \times 3 \mathrm{~cm})$ was transferred to the palm muscle of one subject. The electrical contact with nanosheets was provided by two sheets of Au-sputtered polyimide thin films (thickness: $13 \mathrm{~mm}$ ) placed at both ends of the nanosheet. After the nanosheet and $\mathrm{Au}$ sputtered polyimide thin films were covered with a polyurethane-based transparent adhesive plaster (Cathereeplus ${ }^{\mathrm{TM}}$; Nichiban Co., Ltd, Tokyo), the resistance of the nanosheet was measured at the initial state and the most contracted/stretched state of the palm muscle (Fig. S2a). Even after five repetitive cycles of stretching/contracting, no obvious damage was observed on the nanosheet, and the resistance increased by less than $5 \%$ of the initial value (resistance: from $469 \pm 86$ to $479 \pm 82 \Omega$ ) (Fig. S2b), which was within the range of the standard requirement for the impedance $(<2 \mathrm{k} \Omega)$ of disposable bioelectrodes for the measurement of bioelectric signals, such as an electrocardiogram (according to the American National Standard Institutes, ANSI/AAMI EC12:2000/(R) 2015). These demonstrations clearly indicate that the structural integrity and electrical property of the PEDOT: PSS/SBS nanosheet electrode remained even at the maximum strain of the palm muscle and suggest that the proper functions as bioelectrodes would remain even under tens of repetitive cycles of stretching/contracting.

\section{Construction of the elastic kirigami wiring system}

The elastic kirigami wiring system consisted of a conducting layer made of pantograph-like (combination of rectangular-shaped frames and a straight line)-patterned $\mathrm{Cu}$-sputtered polyimide film $(25.3 \mu \mathrm{m}$ thick; $25-\mu \mathrm{m}$-thick polyimide, and 300-nm-thick $\mathrm{Cu}$ ) sandwiched between 
two insulating layers made of kirigami-patterned silicone rubber sheets $(200 \mu \mathrm{m}$ thick each). We used $\mathrm{Cu}$ and polyimide films for the conductive layer because of the relatively low cost and high electrical conductivity of $\mathrm{Cu}$ and the useful mechanical properties of polyimides, such as flexibility and robustness. The layers were stacked via corona-treatment-assisted bonding (see Supplementary Materials for details) to provide a fully insulated trilayered structure $(425.3 \mu \mathrm{m}$ thick in total; Fig. 1d). The thickness and sheet resistance of the $\mathrm{Cu}$ layer were regulated by adjusting the sputtering time (Fig. S3). In this study, we set the sputtering time to $3 \mathrm{~min}$ and prepared a 300-nm-thick $\mathrm{Cu}$-sputtered polyimide film with a sheet resistance of $0.16 \Omega / \square$ as a conductive layer.

We evaluated the mechanical and electrical properties of the wiring system for three different designs, which were prepared by varying two parameters: the width of the narrowest part of the conductive layer $\left(w_{1}=1.0,0.75\right.$, and $0.5 \mathrm{~mm}$ ) and the distance between the slits in the insulating layer $\left(w_{2}=4.0,3.5\right.$, and $3.0 \mathrm{~mm}$ according to $w_{1}=$ 1.0, 0.75, and $0.5 \mathrm{~mm}$, respectively, as shown in Fig. S4). The width of the margin area on the insulating layer where the top and bottom layers were bonded was constant $\left(w_{3}\right.$ $=0.5 \mathrm{~mm}, w_{4}=1.0 \mathrm{~mm}$, see Fig. S4) for all three designs.

\section{Mechanical and electrical properties of the elastic kirigami wiring system}

Owing to the kirigami structure, the wiring could be stretched with a small tensile force applied by hand (Fig. 2a and Movie S1, showing the case of $w_{1}=0.75$ ). Figure $2 \mathrm{~b}$ shows the microscopic images of the elastic kirigami wiring at $25 \%, 100 \%$, and $150 \%$ tensile strain. The bonding between the top and bottom silicone rubber layers was sufficiently strong that the layers did not detach from one other even when the wiring was stretched to a strain of $150 \%$ (see Fig. 2c). These observations clearly indicate that the corona-treatment-based permanent bonding between the top and bottom silicone rubber layers was strong enough to avoid detachment even under $150 \%$ tensile strain, and thus the conductive layer was completely insulated during kirigami-based 2D-to-3D conversion.

The elastic kirigami wiring system showed hybrid properties of kirigami-based stretchability and siliconerubber-based elasticity. We evaluated the mechanical properties of the wiring system using a tensile tester (Movie S2) and compared the obtained results to theoretical data obtained from a 3D FEM simulation. As displayed in Fig. 2d, the elastic kirigami wiring system showed unique stress-strain (s-s) curves with the following features. First, compared with the control samples ("Nonkirigami" and "Silicone rubber sheet"), a much smaller tensile force $(<0.3 \mathrm{MPa})$ was needed to stretch the wiring system. For example, from the initial slope of the $\mathrm{s}-\mathrm{s}$ curve, that is, at a strain ranging from 0 to $50 \%$,
Young's modulus of the elastic kirigami wiring sample with $w_{1}=0.75$ was calculated to be $15 \mathrm{kPa}$, which is approximately 20,000- and 30-fold smaller than that of the nonkirigami wiring sample $(\sim 313 \mathrm{GPa})$ and that of the silicone rubber sheet $(475 \mathrm{kPa})$, respectively. Second, the elastic kirigami wiring system showed extra stretchability (e.g., $150-200 \%$ strain for the sample with $w_{1}=0.75$ ) even after the end of the kirigami-based 2D-to-3D structural conversion (see the parts of the s-s curves that are jagged just before the wiring completely tore off). The jagged $s-s$ curves were the result of tearing in the insulating layer, which was made of a silicone rubber sheet, at the slits before the conductive layer completely tore off. This is displayed in Movie S2. Figure 2e and Movie S3 show the FEM-simulated deformation of sample $w_{1}=0.75$ at $0 \%$, $50 \%, 100 \%$, and $150 \%$ tensile strain. Although the FEM results could not simulate the rupture of the wiring, including partial tearing of the insulating layer, they matched the experimental data in terms of the range of strain when the wiring was stretched via kirigami-based 2D-to-3D structural conversion.

As shown in Fig. S5a and b, the stretchability and the resistance of the wiring systems, respectively, were modulated by varying the design (the widths $w_{1}$ and $w_{2}$ ). Wiring with $w_{1}=0.75$ produced a resistance of $\sim 6.9 \Omega$ with a smaller error bar than that of $w_{1}=0.5$ and stretchability over $150 \%$ without any structural destruction. The larger error bar of the sample with $w_{1}=0.5$ is assumed to be derived from the damage (i.e., burning) of the $\mathrm{Cu}$ layer caused by the laser cutting process. The narrower width of the wiring would result in larger damage to the $\mathrm{Cu}$ layer, which affects the resistance. Thus, we selected the sample with $w_{1}=0.75$ for further electrical evaluation and applications. Figure 3 a shows the relationship between the applied strain and the normalized resistance $R / R_{0}$ or the loaded tensile stress. In this experiment, we evaluated the sealed (sandwiched between two insulating layers) and bare (only a conductive layer) samples to demonstrate the higher mechanical stability of the sealed sample. For both the sealed and bare samples, the resistance did not change until the wiring was torn off. The results demonstrated that the conductive layer of the kirigami wiring system was stretched via kirigami-based 2D-to-3D structural conversion and that the tensile force was not applied to the materials themselves. The sealed sample exhibited higher stretchability and was conductive at a higher level of strain compared with the bare sample. The conductive layer of the sealed sample was not torn even after it was stretched with over $150 \%$ strain. This is likely due to the presence of the insulating layers made of silicone rubber. In the range where the $s-s$ curves were jagged just before the wiring tore off, we observed damage only in the insulating layer. The conductive layer was not damaged until the wiring was completely ruptured at a 
strain of $\sim 200 \%$ (see Movie S2). The resistance of the sealed wiring remained constant during elongation of the wiring up to $150-200 \%$ tensile strain and then suddenly and drastically increased at the time of rupture.

The sealed sample showed better shape recovery compared with the bare sample due to the elasticity of the silicone rubber (Fig. 3b). As shown in Fig. S6 and Movie S4, the sealed sample did not change its length even after elongation and contraction, while the bare sample deformed (Movie S5) and increased in length (1.3-fold) due to the lack of elasticity. The resistance of the sealed sample did not change during stretching to $150 \%$ strain and contracting to the original length (Fig. 3c).

We also evaluated the electrical durability of the sealed sample for a repeated cycle of stretching and contracting (from 0 to $150 \%$ strain, tensile speed: $3.3 \mathrm{~mm} / \mathrm{s}$ ) (Fig. $3 \mathrm{~d}$ and Movie S6). The resistance of the sample was less than 1.1-fold $(7.5 \Omega)$ and 1.9-fold $(13 \Omega)$ the initial value $(6.9 \Omega$ ) after 10 (Fig. 3e) and 100 cycles (Fig. 3f), respectively. This increase in the resistance of the wiring was derived from some cracks of the $\mathrm{Cu}$ layer at the inner side of the corners, where the mechanical stress was applied the most while the wire was stretched (see images in Fig. 3f). To improve the adhesion between the $\mathrm{Cu}$ layer and the polyimide film as well as the stability of the sputtered $\mathrm{Cu}$ layer, it may be possible to modify the surface of the polyimide before sputtering, for instance, using a surface treatment with corona discharge and/or applying a coating of primers (e.g., silane coupling agents) to impart functional chemical groups that are able to chelate metals ${ }^{35,36}$. Despite the increase in resistance after repeated cycles of stretching and contracting, we demonstrated that a pair of wirings having over 3.0-fold (21.0 and $24.2 \Omega$ ) the initial value could be used for the sEMG measurement on the APBM with a similar SNR to that of another pair of wirings having a resistance of 9.1 and $9.4 \Omega$ (approximately 1.3 -fold the initial value) (Fig. S7). These demonstrations suggest that at least a 3.0-fold increase in the resistance of the wirings caused by the repeated cycle of stretching and contracting did not compromise the efficiency of the sEMG signals. According to the American National Standards Institute for disposable electrodes (ANSI/AAMI EC12:2000/(R)2015), the impedance of the electrodes should not exceed $2 \mathrm{k} \Omega$. Considering that the resistance of the conductive nanosheet is approximately $0.5 \mathrm{k} \Omega$ (from the measured sheet resistance), the resistance of the wirings should be lower than $1.5 \mathrm{k} \Omega$ to make the combined resistance within the required range. Judging from the result shown in Fig. 3f, it seems that more than 10000 cycles were required for the resistance to reach $1.5 \mathrm{k} \Omega(\sim 200$-fold the initial value). This estimation suggests that the samples after at least thousands of cycles of elongation/contraction could be used for the sEMG measurement.
To demonstrate that the insulating property of the elastic kirigami wiring system was due to the combination of the pantograph-like conductive layer and the kirigamipatterned insulating layers, we compared four different samples: "sealed" (consisting of a pantograph-like conductive layer sandwiched between kirigami-patterned insulating layers), "semisealed A" (consisting of a kirigami-patterned conductive layer sandwiched between the same kirigami-patterned insulating layers), "semisealed B" (consisting of a pantograph-like conductive layer sandwiched between the same pantograph-like insulating layers), and "bare" (consisting of a pantograph-like conductive layer without insulating layers). "Semisealed" means that only the edge of the conductive layer was exposed. The illustrations and microscopic images of these samples are shown in Fig. 4a, b, respectively. Notably, the impedance of the sealed sample (over $10 \mathrm{M} \Omega$ at and between 50 and $150 \mathrm{~Hz}$, which is the most dominant range of $\mathrm{EMG}^{37}$, and overload at $<50 \mathrm{~Hz}$ ) did not decrease even when the wiring was stretched at $150 \%$ strain, while the other three samples exhibited low impedance $(<10 \mathrm{k} \Omega$ in the range from 50 to $150 \mathrm{~Hz})$ (Fig. 4c). These results indicate that the conductive layer was fully insulated by the silicone rubber layers and that the bonding between the top and bottom layers was sufficiently strong that these layers did not detach even when stretched at $150 \%$ strain.

\section{Dynamic sEMG analysis of the APBM during baseball pitching}

Finally, we assembled a device consisting of elastic kirigami wiring and conductive nanosheets termed the "elastic kirigami patch" (Fig. 1a). The edge of the elastic kirigami wirings, where the $\mathrm{Cu}$ layer was exposed, was flat and fixed on the conducive nanosheet electrode using Cathereeplus ${ }^{\mathrm{TM}}$ to avoid a change in the distance between the copper and the palm during the motion of the arm. The kirigami part of the wiring was not covered by Cathereeplus $^{\mathrm{TM}}$ (see Fig. 1a), i.e., not fixed to the skin. Additionally, as shown in Fig. 4c, the conductive layer of the wirings was completely insulated by the $200-\mu \mathrm{m}$-thick silicone rubber. Thus, the effect of the change in distance between the kirigami part of the wirings and the skin caused by out-of-plane tilting of the kirigami structure during stretching on the measured sEMG signals was estimated to be negligible. We measured sEMG signals from the right hand and arm of two participants: an experienced baseball player ("participant A", male, age: 18 , height: $5.64 \mathrm{ft}$, weight: $143 \mathrm{lbs}$, right-handed, overhand throw, years of baseball experience: 8) and a nonexperienced person ("participant B", male, age: 36 , height: $5.74 \mathrm{ft}$, weight: $165 \mathrm{lbs}$, right-handed, overhand throw). To record sEMG from the palm muscle (APBM), we fabricated a patch by assembling two elastic kirigami wiring 


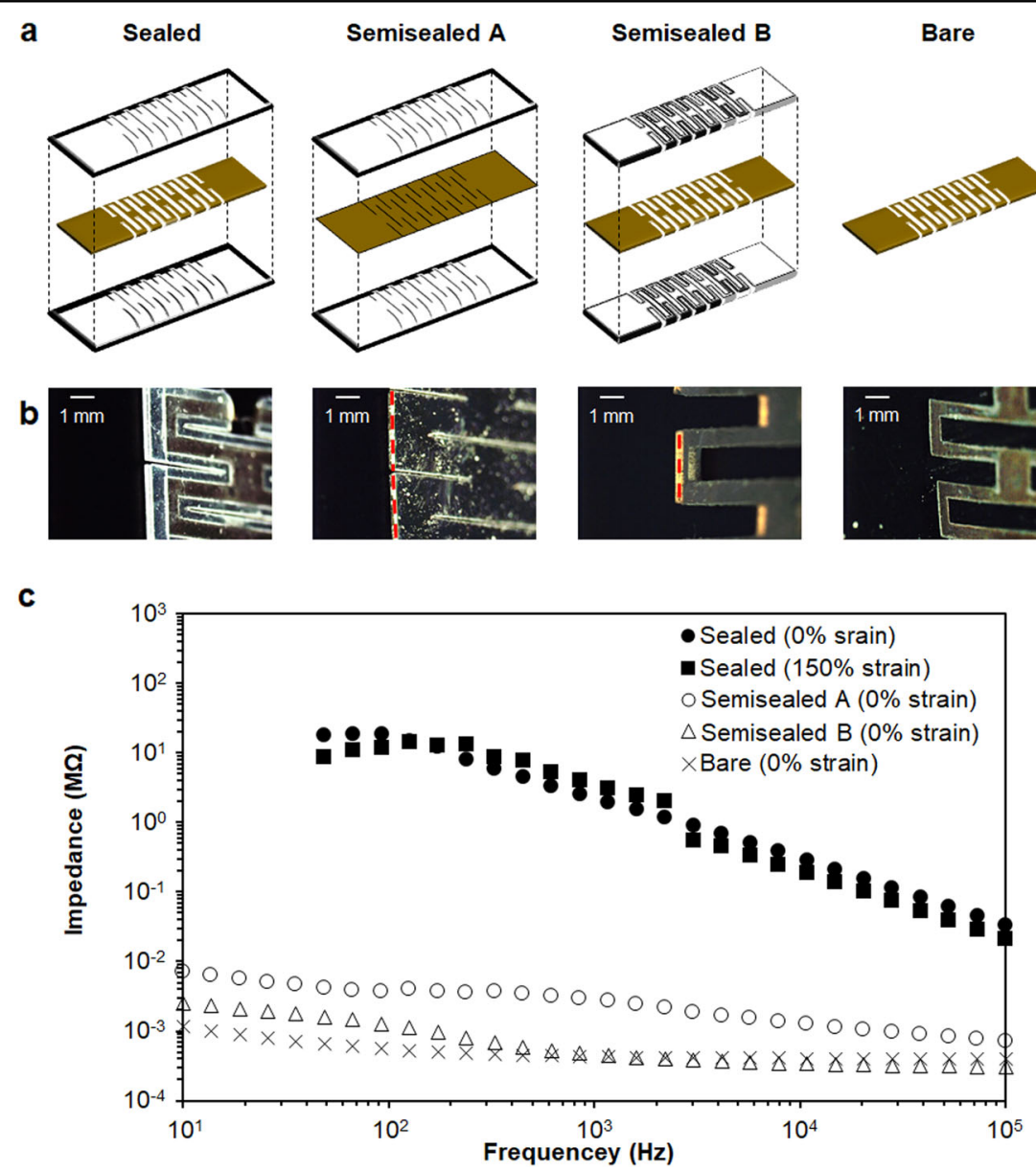

Fig. 4 Insulation property of elastic kirigami wiring. a Schematic illustration of the design for sealed, semisealed A, semisealed B, and bare (left to right) samples. $\mathbf{b}$ Microscopic images of sealed, semisealed A, semisealed B, and bare (left to right) samples. The red dashed line in the middle image represents the exposed conductive layer. c Relationship between the frequency and impedance of the sealed, semisealed A, semisealed B, and bare samples. For the sealed sample, the impedance was measured for the initial length ( $0 \%$ strain) and elongated state (150\% strain).

structures on Cathereeplus ${ }^{\mathrm{TM}}$. As ultraconformable skincontact electrodes, we transferred two conductive nanosheets onto the thenar area of the palm of the participant (see Fig. S1 for methods). We then attached the edges of the elastic kirigami wiring structures (with the $\mathrm{Cu}$ layer exposed) onto the nanosheets to obtain an electrical connection between the electrodes and the wiring system (Fig. S8a). The APBM, as illustrated in Fig. S8b, functions as an abductor of the thumb. Thus, the sEMG signal from the APBM represents the force used to grasp the ball during pitching.

To evaluate the mechanical and electrical stability of the skin-contact device consisting of the elastic kirigami patch and a Bluetooth module during pitching, we measured the electrode-skin contact impedance before and after the participants performed an arm swing and compared these data with the impedance measured with a nonkirigami sample (see Fig. S3 for the composition of the nonkirigami sample). As a result, the impedance of the kirigami patch
$(<100 \mathrm{k} \Omega$ at $50-150 \mathrm{kHz})$ did not change even after swinging the arm in a pitching-like motion (maximum acceleration of the arm: $\sim 20 \mathrm{~m} / \mathrm{s}^{2}$ ), while that of the nonkirigami patch increased to $>100 \mathrm{k} \Omega$ due to the rupture and detachment of the nanosheet electrodes from the skin (Fig. S9). This demonstration strongly suggested that the kirigami structure of the wiring played a critical role in buffering the mechanical stress applied to the electrodes during the highly accelerated swing. Although the participant swung the arm without holding the ball in this experiment, the conductive nanosheets and the edge of the elastic kirigami wirings were covered and protected by Cathereeplus ${ }^{\mathrm{TM}}$, which can prevent the detachment or damage of the device caused by the friction between the ball and skin during pitching.

To investigate differences in the sEMG signal pattern between the APBM and other muscles, we equipped the arm of the participant with two wireless and cable-less EMG sensors (PicoEMG; Cometa Systems, Bareggio). 
This enabled us to record sEMG signals from two muscles in the forearm-the flexor carpi ulnaris muscle (FCUM) and the extensor carpi radialis longus muscle (ECRLM)using standard $\mathrm{Ag} / \mathrm{AgCl}$ pre-gelled electrodes (Fig. S8c). The FCUM and ECRLM, illustrated in Fig. S8d, function to adduct and abduct the hand at the wrist, respectively. Thus, these two muscles are antagonistic muscles.

We used three high-speed cameras to capture the pitching motion of the participants. Specifically, we obtained a sequence of photographs of the participant's arm and hand during the release of the ball. The speed of each pitched ball (unit: miles per hour, MPH) was measured via a speed gun (CR-1K, JUGS, Oregon). Data regarding the sEMG signals, which were recorded by three modules, and the acceleration of the wrist $(x, y$, and $z$ directions), which was recorded by a module secured to the wrist and connected to the patch, were transmitted to a laptop computer via Bluetooth wireless communication in real-time during pitching.

Participant A pitched three fastballs and three curveballs and participant B pitched three fastballs. From the photograph sequence of pitching motion obtained by the high-speed camera (Movies S7-9), time $t=0 \mathrm{~s}$ was defined as the point of ball release. We used three devices (the elastic kirigami patch with the Bluetooth module for the APBM and the two gel-based EMG sensors for the FCUM and ECRLM) to record SEMG signals from $t=$ -2.0 to $0.2 \mathrm{~s}$ (Figs. S10a, S11a, and S12a, which represent participant A's fastball, B's fastball, and A's curveball, respectively). Simultaneously, the acceleration profiles (three-axis composite value) were also recorded by a module connected to the elastic kirigami patch (the sEMG sensor and accelerator were built into one module). Photograph sequences of the pitching motion are shown in Fig. S10b (participant A's fastball), S11b (participant B's fastball), and S12b (participant A's curveball). The representative sEMG and acceleration data for the fastball (ball speed: $68 \mathrm{MPH}$ ) and curveball (57 MPH) thrown by participant A are shown in Fig. 5a, b, respectively.

The data indicated that participant A used the APBM to grasp the fastball from the initial motion of pitching until ball release. The sEMG intensity of the APBM in participant A began to gradually increase at $t=\sim-0.6 \mathrm{~s}$ (see Fig. S10), while the sEMG signal of participant B drastically increased at $t=\sim-0.1 \mathrm{~s}$, that is, just before the release of the ball (see Fig. S11). This suggests that participant A grasped the ball to prevent the ball from slipping out of their hand during the initial pitching phases, while participant B did not use the APBM to grasp the ball as strongly as participant A and applied force only just before releasing the ball. The higher maximum acceleration of the arm in participant A $\left(247 \pm 9 \mathrm{~m} / \mathrm{s}^{2}\right)$ and the larger MER angle compared with participant $\mathrm{B}$ (maximum acceleration: $230 \pm 34 \mathrm{~m} / \mathrm{s}^{2}$ ) also support this notion.
For a more quantitative analysis of the sEMG signals, the obtained sEMG data were normalized to the maximum voluntary contraction (MVC), which was measured by taking the maximal isometric exertion of each muscle against a fixated static resistance ${ }^{38}$. We obtained the MVC for each muscle in each participant (only the data for participant A are shown in Fig. S13) using the same patch and EMG sensors as those used in the pitching measurements. The SNR for each recorded sEMG was calculated. The SNR of the sEMG signal on the APBM $(35.1 \mathrm{~dB})$ recorded by using the elastic kirigami patch compared favorably with those on the FCUM $(40.9 \mathrm{~dB})$ and ECRLM (37.4 $\mathrm{dB}$ ) recorded by using the standard $\mathrm{Ag} /$ $\mathrm{AgCl}$ pre-gelled electrodes. These results indicate that our sEMG measurement system has comparable sensitivity with that of the conventional standard method.

The patterns of the obtained sEMG signals from the FCUM and ECRLM for all pitching trials were similar to previously reported values, which were recorded from a baseball pitcher using wearable wireless sEMG sensors and $\mathrm{Ag} / \mathrm{AgCl}$ bioelectrodes ${ }^{39}$. Notably, because of the flexibility, stretchability, and durability of the elastic kirigami wiring system, the patch, Bluetooth module, and two EMG sensors remained secure on the skin without detachment or rupture during the trials. This was the case even when the wrist joint was dorsiflexed before releasing the ball and given that the maximum acceleration of the wrist during the fastball pitch was $247 \pm 9 \mathrm{~m} / \mathrm{s}^{2}(n=3)$ at the time of ball release. Thus, we obtained sEMG and acceleration signals from the APBM as well as the FCUM and ECRLM throughout the motion of the pitch.

Figure $5 \mathrm{c}, \mathrm{d}$ shows images of the pitching motion as participant A threw a fastball and a curveball, respectively. The figures show the hand holding the ball $(t=\sim$ $-100 \mathrm{~ms}$ ), the upper arm at the maximum point of shoulder external rotation (MER, $t=-50-40 \mathrm{~ms}$ ), and the upper arm at the point of ball release $(t=0)$. Comparing the fastball and curveball trials of participant $\mathrm{A}$, we observed differences in the position of the thumb and the rotation angle of the forearm at ball release. Specifically, the thumb was farther from the pinky finger, and the forearm was more supinated when pitching curveballs, despite no difference in the MER angle.

The pitching motions were separated into five phases: wind-up, early cocking, late cocking, acceleration, and follow-through (Fig. 5e, f for the fastball and curveball trials, respectively $)^{40}$. The timing and period of each phase was almost the same between the two different types of pitches. Figure $5 \mathrm{~g}$ shows the mean sEMG signal from the APBM, normalized to the MVC, for the two different kinds of pitch trial (fastball and curveball performed by participant A, with $n=3$ for each pitch). The $\% \mathrm{MVC}$ signal of the fastball trials had peaks at $t=-1.4$ to $-1.1 \mathrm{~s}$ (early stage of the wind-up) and $t=-0.5$ to $0.1 \mathrm{~s}$ (early 


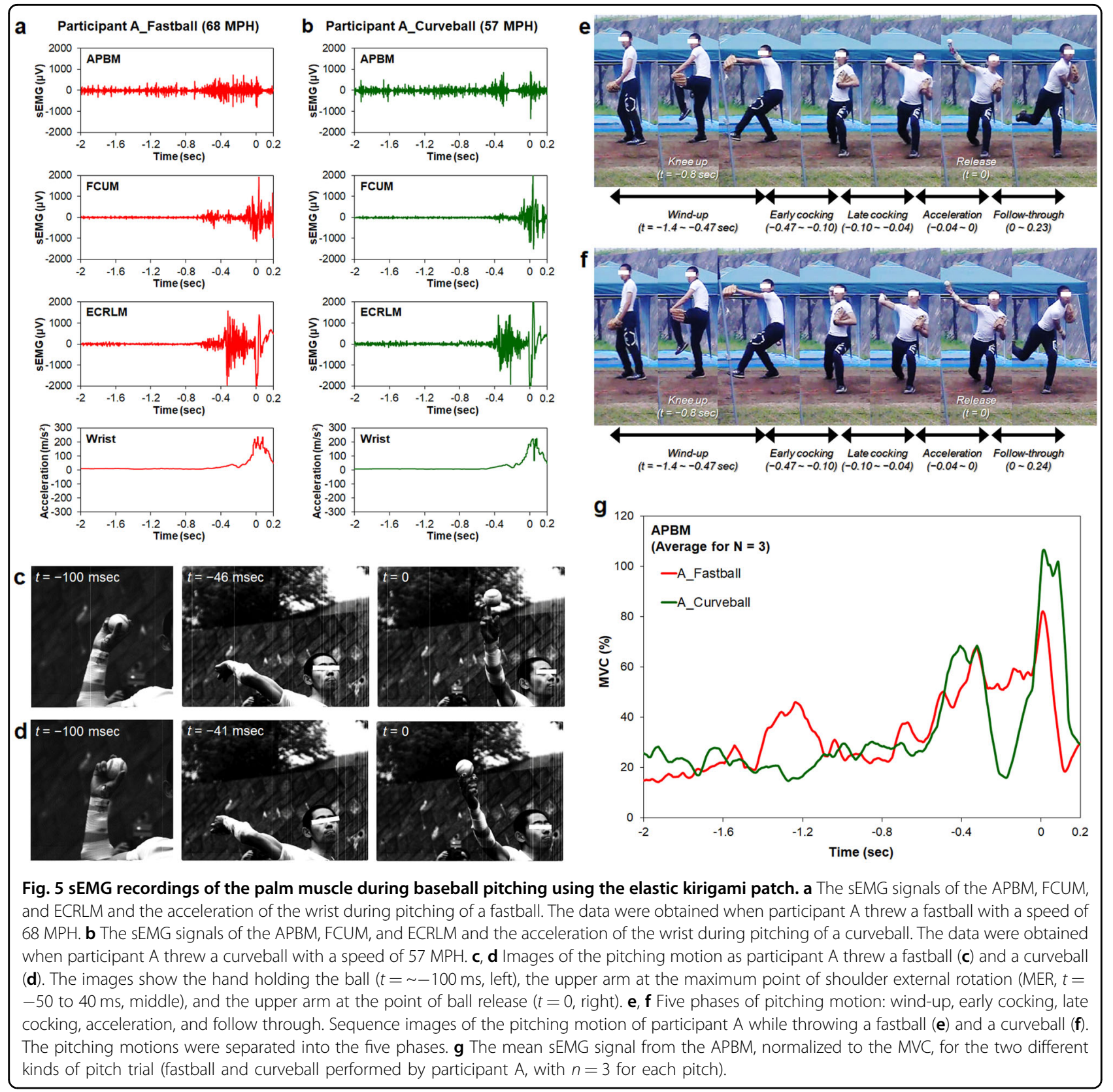

cocking). That of the curveball did not have a peak around $t=-1.4$ to $-1.1 \mathrm{~s}$ but instead showed separated peaks at $t=-0.5$ to $-0.3 \mathrm{~s}$ and $t=-0.03$ to $0.1 \mathrm{~s}$, that is, a drop in intensity at $t=-0.3$ to $-0.03 \mathrm{~s}$ (early cocking-late cocking). Although conventional technologies such as motion capture systems and high-speed videos have already been used to characterize how pitchers adjust the orientation of their hand at ball release when throwing breaking balls such as curveballs ${ }^{41}$, the dynamic electromyographic pattern of palm muscle activity has not been reported due to the lack of suitable methods for measuring sEMG on the palm during pitching. We observed differences in APBM activity between the fastball and curveball trials, although no differences were present between the FCUM and ECRLM activity (Fig. S14a and b, respectively). These differences are likely associated with the way the ball is held in the fingers and thrown, including the angle of the wrist, and may represent the characteristic muscle activity necessary to keep grasping the ball when the position of the thumb changes depending on the intended type of throw (e.g., fastball vs. curveball). Generally, it is more difficult for pitchers to control curveballs compared with fastballs; this may be related to the strengthening and weakening of APBM activity after $t=-0.5 \mathrm{~s}$ when throwing curveballs. Our electromyographic analyses of the APBM during the whole pitching motion using the 
elastic kirigami patch indicate that pitchers control the muscle activity of the palm even in the early cocking phase, that is, before ball release.

\section{Conclusions}

We developed a skin-contact patch device consisting of a kirigami-inspired stretchable wiring system and conductive nanosheet-based ultraconformable bioelectrodes. We successfully conducted dynamic sEMG analysis of the APBM, where conventional devices could not be applied, during baseball pitching. The advantages of patches using the elastic kirigami wiring system in wearable applications are as follows: (i) they enable contact of the skin surface as a 2D membrane, offering a stable electrical connection from skin-contacted conductive nanosheet electrodes; (ii) they require minimal force to be stretched, leading to minimal mechanical stress to the nanosheet-based bioelectrodes during exercise; and (iii) the conductive region is fully insulated so that it is protected from sweat or other external factors.

The elastic kirigami patch, with a minimal tensile stress requirement for stretching and over $150 \%$ stretchability, is an effective solution for the mechanical mismatch between electrical devices and the human body. When we combined the patches with conductive polymer nanosheets and Bluetooth-based electromyography, we succeeded in precisely recording SEMG at the APBM during pitching. We observed different APBM sEMG patterns between fastball and curveball throws in an experienced baseball player. We believe that the conductive nanosheets and the elastic kirigami patch will serve as one of the minimally perceivable devices to investigate the activity of any muscles of athletes during exercise without interfering with their performance. Moreover, our sEMG measurement system will enable the analysis of motion in unexplored muscle areas, such as on the palm and the sole, leading to a deeper understanding of muscular activity during the performance in a wide range of sports and other movements.

\section{Acknowledgements}

This work was supported by the Precursory Research for Embryonic Science and Technology (PRESTO) from the Japan Science and Technology Agency (JST; grant number JPMJPR152A), JSPS KAKENHI (grant number 17K20116, 18H03539, 18H05469), Private University Research Branding Project: Social Design on Medical, Sci-Tech and Sociology (Waseda University), MEXT Japan, Human Performance Laboratory, Organization for University Research Initiative, Waseda University, the Noguchi Institute, the Tanaka Memorial Foundation, and the Terumo Foundation for Life Sciences and Arts. T.F. is supported by the Leading Initiative for Excellent Young Researchers (LEADER) by MEXT, Japan. The authors would like to thank Ms. Marin Okamoto for her assistance with painting the figures (Fig. S8).

\section{Author details}

'Research Organization for Nano \& Life Innovation, Waseda University, Tokyo, Japan. ${ }^{2}$ Graduate School of Advanced Science and Engineering, Waseda University, Tokyo, Japan. ${ }^{3}$ Waseda Research Institute for Science and
Engineering, Waseda University, Tokyo, Japan. ${ }^{4}$ Faculty of Science and Engineering, Waseda University, Tokyo, Japan. ${ }^{5}$ Faculty of Sport Sciences, Waseda University, Saitama, Japan. ${ }^{6}$ Center for Human and Social Sciences, Kitasato University College of Liberal Arts and Sciences, Kanagawa, Japan. ${ }^{7}$ Japan Science and Technology Agency, PRESTO, Saitama, Japan. ${ }^{8}$ School of Life Science and Technology, Tokyo Institute of Technology, Kanagawa, Japan. ${ }^{9}$ Present address: Digital Manufacturing and Design (DManD) Centre, Singapore University of Technology and Design, Singapore, Singapore

\section{Authors' contributions}

T. Nagami and T.F. jointly supervised this work. K.Y. and T. Nakanishi prepared and characterized the conductive nanosheets. K.Y., T. Nakanishi, S.M., and M.A. prepared and characterized the elastic kirigami wirings. K.Y., T. Nakanishi, S.M. T. Nagami, and T.F. carried out the sEMG measurement on participants, and K.Y and T. Nagami analyzed the obtained data. K.Y. wrote the manuscript under the supervision and guidance of S.T., K.K., T. Nagami, and T.F. All authors discussed the results and commented on the manuscript.

\section{Data availability}

The authors declare that all data supporting the findings of this study are available within the paper and its Supplementary Information as well as from the corresponding authors upon reasonable request.

Conflict of interest

The authors declare that they have no conflict of interest.

\section{Publisher's note}

Springer Nature remains neutral with regard to jurisdictional claims in published maps and institutional affiliations.

Supplementary information is available for this paper at https://doi.org/ 10.1038/s41427-019-0183-1.

Received: 22 June 2019 Revised: 17 October 2019 Accepted: 25 October 2019.

Published online: 12 December 2019

\section{References}

1. Kim, D.-H. et al. Epidermal electronics. Science 333, 838-844 (2011).

2. $\mathrm{Xu}, \mathrm{B}$. et al. An epidermal stimulation and sensing platform for sensorimotor prosthetic control, management of lower back exertion, and electrical muscle activation. Adv. Mater. 28, 4462-4471 (2016).

3. $\mathrm{Xu}, \mathrm{S}$. et al. Soft microfluidic assemblies of sensors, circuits, and radios for the skin. Science 344, 70-74 (2014).

4. Son, D. et al. Multifunctional wearable devices for diagnosis and therapy of movement disorders. Nat. Nanotechnol. 9, 397-404 (2014).

5. Jeong, J. et al. Materials and optimized designs for human-machine interfaces via epidermal electronics. Adv. Mater. 25, 6839-6846 (2013).

6. Stevenson, J. M. Finger release sequence for fastball and curveball pitches. Can. J. Appl. Sport Sci. 10, 21-25 (1985).

7. Hore, J., Watts, S., Martin, J. \& Miller, B. Timing of finger opening and ball release in fast and accurate overarm throws. Exp. Brain Res. 103, 277-286 (1995).

8. Hore, J., Watts, S., Hore, J. \& Watts, S. Skilled throwers use physics to time ball release to the nearest millisecond. J. Neurophysiol. 106, 2024-2033 (2011).

9. Nagami, T., Morohoshi, J. \& Kanosue, K. Relationship between ball control and movements of hand and fingers in baseball pitchers. in JSME Joint Symposium 2009, Fukuoka. 51-55 (2009).

10. Wkurzlqj, D. U. P. et al. Disorders in timing and force of finger opening in overarm throws made by cerebellar subjects. Ann. NY Acad. Sci. 978, 1-15 (2002).

11. Fleisig, G. S., Barrentine, S. W., Andrews, J. R., Escamilla, R. F. \& Matsuo, T. Kinematic analysis of the wrist and forearm during baseball pitching. J. Appl. Biomech. 14, 24-39 (1998).

12. Di Giovine, N. M., Jobe, F. W. \& Perry, J. An electromyographic analysis of the elbow in pitching. Am. J. Sports Med. 15, 260-263 (1992). 
13. Henning, L., Plummer, H. \& Oliver, G. D. Comparison of scapular muscle activations during three overhead throwing exercises. Int. J. Sports Phys. Ther. 11, 108-114 (2016).

14. Yamanouchi, T. EMG analysis of the lower extremities during pitching in highschool baseball. Kurume Med. J. 45, 21-25 (1998).

15. DiGiovine, N. M., Jobe, F. W., Pink, M. \& Perry, J. An electromyographic analysis of the upper extremity in pitching. J. Shoulder Elbow Surg. 1, 15-25 (1992).

16. Lee, S. M. et al. Self-adhesive epidermal carbon nanotube electronics for tether-free long-term continuous recording of biosignals. Sci. Rep. 4, 6074 (2014).

17. Pang, C., Lee, C. \& Suh, K. Y. Recent advances in flexible sensors for wearable and implantable devices. J. Appl. Polym. Sci. 130, 1429-1441 (2013).

18. Huang, $X$. et al. Materials and designs for wireless epidermal sensors of hydration and strain. Adv. Funct. Mater. 24, 3846-3854 (2014).

19. Yamagishi, K., Takeoka, S. \& Fujie, T. Printed nanofilms mechanically conforming to living bodies. Biomater. Sci. 7, 520-531 (2019).

20. Matsuhisa, N. et al. Printable elastic conductors with a high conductivity for electronic textile applications. Nat. Commun. 6, 7461 (2015).

21. Matsuhisa, N. et al. Printable elastic conductors by in situ formation of silver nanoparticles from silver flakes. Nat. Mater. 16, 834-840 (2017).

22. Tada, T., Inoue, M. \& Tokumaru, T. A characteristic evaluation of an undershirt for measurement of bioelectricity using conductive ink wires. J. Text. Eng. 59 141-148 (2013).

23. Stoppa, M. \& Chiolerio, A. Wearable electronics and smart textiles: a critical review. Sensors 14, 11957-11992 (2014).

24. Zhai, Y., Liu, X., Wang, Y. \& Li, X. Progress in preparation and application of fabric electrodes. J. Miner. Mater. Charact. Eng. 2, 598-604 (2014).

25. Kokubo, N. et al. Inkjet-printed neural electrodes with mechanically gradient structure. ACS Appl. Bio Mater. 2, 20-26 (2019).

26. Blees, M. K. et al. Graphene kirigami. Nature 524, 204-207 (2015).

27. Shyu, T. C. et al. A kirigami approach to engineering elasticity in nanocomposites through patterned defects. Nat. Mater. 14, 785-790 (2015).
28. Xu, L., Shyu, T. C. \& Kotov, N. A. Origami and kirigami nanocomposites. ACS Nano 11, 7587-7599 (2017).

29. Morikawa, Y., Yamagiwa, S., Sawahata, H., Numano, R. \& Koida, K. Ultrastretchable kirigami bioprobes. Adv. Healthc. Mater. 7, 1701100 (2018).

30. Zhao, R., Lin, S., Yuk, H. \& Zhao, X. Kirigami enhances film adhesion. Soft Matter 14, 2515-2525 (2018).

31. Zucca, A. et al. Roll to roll processing of ultraconformable conducting polymer nanosheets. J. Mater. Chem. C 3, 6539-6548 (2015).

32. Yamagishi, K. et al. in Flexible and Stretchable Medical Devices (ed. Takei, K.) 253-283 (Wiley-VCH Verlag GmbH \& Co. KGaA, 2018).

33. Tetsu, Y. et al. Ultrathin epidermal strain sensor based on an elastomer nanosheet with an inkjet-printed conductive polymer. Appl. Phys. Express 10, 087201 (2017).

34. Sato, N., Murata, A. \& Takeoka, S. Stretchable, adhesive and ultra-conformable elastomer thin films. Soft Matter 12, 9202-9209 (2016).

35. Iwamura, E. Improvement of adhesion strength in thin films. J. Surf. Finish. Soc. Jpn. 58, 260-266 (2007)

36. Kinbara, A. Science and technology of thin film adhesion. J. Surf. Finish. Soc Jpn. 63, 710-717 (2012).

37. Samarawickrama, K. et al. Surface EMG signal acquisition analysis and classification for the operation of a prosthetic limb. Int. J. Biosci. Biochem. Bioinformatics 8, 32-41 (2018).

38. Al-Qaisi, S. \& Aghazadeh, F. Electromyography analysis: comparison of maximum voluntary contraction methods for anterior deltoid and trapezius muscles. Procedia Manuf. 3, 4578-4583 (2015).

39. Hirashima, M., Technology, C., Kadota, H., Kudo, K. \& Ohtsuki, T. Sequentia muscle activity and its functional role in the upper extremity and trunk during overarm throwing. J. Sports Sci. 20, 301-310 (2002).

40. Jobe, F. W., Moynes, D. R., Tibone, J. E. \& Perry, J. An EMG analysis of the shoulder in pitching. Am. J. Sports Med. 12, 218-220 (2007).

41. Nagami, T., Higuchi, T. \& Kanosue, K. in Sports Performance (eds. Kanosue, K, Nagami, T. \& Tsuchiya, J.) 323-333 (Springer, Japan, 2015). 